\title{
Assessment of levels of D-dimer and interferon- $\gamma$ in pediatric patients with Mycoplasma pneumoniae pneumonia and its clinical implication
}

\author{
XIAOQIU JIN ${ }^{1}$, YING ZHU ${ }^{2}$, YINGCHUN ZHANG ${ }^{3}$, JING CHEN $^{2}$, LI RONG $^{4}$ and XUESONG ZHAO ${ }^{5}$ \\ ${ }^{1}$ Teaching and Research Office of Pediatrics; Departments of ${ }^{2}$ Pediatrics and ${ }^{3}$ Clinical Laboratory, \\ The Second Affiliated Hospital of Qiqihar Medical University, Qiqihar, Heilongjiang 161000; ${ }^{4}$ Department of Pediatrics, \\ The First Hospital of Qiqihar City, Qiqihar, Heilongjiang 161006; ${ }^{5}$ Clinical Laboratory, The Second Affiliated Hospital of \\ Qiqihar Medical University, Qiqihar, Heilongjiang 161000, P.R. China
}

Received May 24, 2018; Accepted September 24, 2018

DOI: $10.3892 /$ etm.2018.6873

\begin{abstract}
Levels of D-dimer and interferon- $\gamma$ (INF- $\gamma$ ) in pediatric patients with Mycoplasma pneumoniae pneumonia (MPP) were assessed to explore its clinical implication in evaluating the disease severity and patients' prognosis. Enrolled in this study as subjects were 185 pediatric patients with MPP who were admitted to the Department of Pediatrics of the Second Affiliated Hospital of Qiqihar Medical University and the First Hospital of Qiqihar City from January 2017 to October 2017 and 115 healthy children who underwent physical examination during the same time period. Patients were divided into two groups according to the severity of their pneumonia: Severe pneumonia group with 95 patients and mild pneumonia group with 90 patients. A total of 92 healthy children were assigned to the control group. Immunoassay was performed to measure the serum levels of D-dimer and INF- $\gamma$. The levels of these two markers were compared. In terms of D-dimer, the levels in pediatric patients in pneumonia groups were all higher than that in the healthy children $(\mathrm{p}<0.0001)$. After treatment for $120 \mathrm{~h}$, the D-dimer and INF- $\gamma$ levels in both pneumonia groups were lower than those before treatment $(\mathrm{p}<0.001)$. Among the patients in both the mild and severe pneumonia groups, those who developed extra-pulmonary complications had higher levels of D-dimer and INF- $\gamma$ than those who did not developed extra-pulmonary complications $(\mathrm{p}<0.001)$. Increased levels of D-dimer and INF- $\gamma$ were observed in pediatric patients with MPP. Assessment of the levels of D-dimer and INF- $\gamma$ in serum may help with the diagnosis of severe MPP in children and predicting the
\end{abstract}

Correspondence to: Dr Xiaoqiu Jin, Teaching and Research Office of Pediatrics, The Second Affiliated Hospital of Qiqihar Medical University, 37 Zhonghua West Road, Qiqihar, Heilongjiang 161000, P.R. China

E-mail: qje2h6@163.com

Key words: pneumonia in children, Mycoplasma pneumoniae pneumonia, D-dimer, interferon- $\gamma$ occurrence of complications. This study provided more theoretical basis for the early diagnosis and identification of MPP in children, and can be used as a guideline in evaluating the severity and preventing complications.

\section{Introduction}

Pneumonia is a common respiratory disease in the clinic. It is an inflammation of the alveoli, the distal airways, and interstitium of the lungs caused by infection of pathogenic microorganisms, immune regulation, physical and chemical factors. The main clinical manifestations include fever, cough, shortness of breath and continuous dry and wet rales in the lung (1). Mycoplasma pneumoniae pneumonia (MPP) is the most common form of pneumonia in childhood caused by Mycoplasma pneumoniae (MP) infection. In China, MPP is a leading cause of death in children (2). In recent years, the number of pediatric patients infected with MP has increased year by year worldwide, and patient age has shown a younger trend (3). However, the diagnosis or differential diagnosis of MPP is difficult based on clinical symptoms. In this regard, the lab test is especially helpful in the diagnosis of MPP. Although D-dimer and interferon- $\gamma(\mathrm{INF}-\gamma)$ were reported to be associated with pneumonia in the literature, the diagnostic value and clinical implication of assessing both indicators in diagnosis of MPP in children were not reported (4-7). In this study, the serum levels of D-dimer and INF- $\gamma$ in 185 pediatric patients with MPP and 92 healthy children were measured, to explore their associations with MPP and the clinical value of assessing both in diagnosis of MPP. The aim of this study was to find a more accurate, convenient and rapid diagnostic method for the clinical prediction and diagnosis of MPP in children.

\section{Patients and methods}

Subjects. Enrolled in this study were 185 pediatric patients with MPP confirmed through assessment of clinical presentation, medical history, chest X-ray radiography findings and laboratory data who were admitted to the Second Affiliated 
Hospital of Qiqihar Medical University's Pediatric Department and the First Hospital of Qiqihar City (both in Qiqihar, China) from January 2017 to October 2017. Patients were divided into two groups according to the severity of their sickness: The mild and severe pneumonia group with an average age of $3.316 \pm 1.088$ and $3.350 \pm 1.078$ years, respectively. All patients met the criteria for the diagnosis of MPP outlined in Zhu Fu Tang Practical Pediatrics, including fever duration of $>10$ days, severe clinical presentation in the lungs, pulmonary consolidation and pleural effusion. Patients who developed extra-pulmonary complications or experienced poor outcomes after 10 days of solo treatment with macrolide antibiotics were regarded as being with severe MPP (8). A total of 92 healthy children with an average age of $3.296 \pm 1.127$ years who underwent physical examination during the same time period were assigned to the control group. The general data such as sex and age of the three groups of subjects were comparable, and the differences between groups were not statistically significant. Patients with the following conditions were excluded from this study: Diarrhea, anemia, liver and kidney diseases, systemic diseases, congenital heart disease, congenital immunodeficiency disorders, long-term use of immunosuppressive agents, tuberculosis and asthma. The duration of fever or cough was 2-7 days for all patients with MPP. This study was approved by the Ethics Committee of the Second Affiliated Hospital of Qiqihar Medical University. All participating children and their families were briefed on the specific protocols and procedures of this study. Guardians of all the children participating in this study signed informed consent and children voluntarily participated in this study.

Sample collection and plasma D-dimer detection. Pre- and post-treatment blood samples were collected from pediatric patients with mild and severe pneumonia. Each time $5 \mathrm{ml}$ of venous blood was taken from all the patients in the morning under fasting. The blood sample was centrifuged at $500 \mathrm{x} \mathrm{g}$ for 10 min using a centrifuge manufactured by Anhui Zhongke Zhongjia Scientific Instrument Co., Ltd. (Hefei, China). The plasma was stored in a dry tube protected from light. The D-dimer level was determined using the erythrocyte sedimentation rate (ESR) test kit (Siemens AG, Munich, Germany) with the immune turbidimetric assay. The assay was performed on a fully automated hematology analyzer (Sysmex Corp., Kobe, Japan). The kit was used strictly in accordance with the manufacturer's protocol. The measured plasma D-dimer levels were recorded for all subjects in the three groups. A D-dimer level $>0.115 \mu \mathrm{g} / \mathrm{ml}$ was regarded as positive.

Serum INF- $\gamma$ detection. Pre- and post-treatment blood samples were collected from all pediatric patients with mild and severe pneumonia. Blood samples were also collected from the healthy subjects. Each time $5 \mathrm{ml}$ of venous blood was taken in the morning under fasting. The blood sample was centrifuged at $500 \mathrm{x}$ g for $10 \mathrm{~min}$ using a centrifuge manufactured by Anhui Zhongke Zhongjia Scientific Instrument Co., Ltd. The supernatant (serum) was taken and stored in a freezer at $-80^{\circ} \mathrm{C}$. The serum level of INF- $\gamma$ was determined with the enzyme-linked immunosorbent assay (ELISA) using the ELISA kit (Thermo Fisher Scientific, Inc., Waltham, MA, USA). The kit was used strictly in accordance with
Table I. General data of subjects in the three groups.

\begin{tabular}{lccc}
\hline & \multicolumn{3}{c}{ Groups } \\
\cline { 2 - 4 } Items & \multicolumn{3}{c}{$\begin{array}{c}\text { Mild } \\
\text { pneumonia }\end{array}$} \\
$\begin{array}{lccc}\text { Severe } \\
\text { pneumonia }\end{array}$ \\
\hline Case no. & 92 & 90 & 95 \\
Sex & & & \\
Male & 46 & 46 & 48 \\
Female & 46 & 44 & 47 \\
Average age (years) & 3.296 & 3.316 & 3.350 \\
Fever (n) & 0 & 90 & 95 \\
Cough (n) & 0 & 90 & 95 \\
$\begin{array}{l}\text { Abnormal findings in } \\
\text { chest X-ray radiography (n) }\end{array}$ & 0 & 90 & 95 \\
Positive responses in & 0 & 90 & 95 \\
MP antibody test (n) & & & \\
\hline
\end{tabular}

MP, Mycoplasma pneumoniae.

the manufacturer's protocol. The assay was performed on a Biotek EL 311 (BioTec U.S., Winooski, VT, USA) microplate reader. The measured serum levels of INF- $\gamma$ were recorded for all subjects in the three groups.

Statistical analysis. All data were expressed as mean \pm SEM. The t-test was used for the comparison of two means. All data were subjected to statistical analysis using the analysis of variance (ANOVA) statistical software. Fisher's analysis was used after ANOVA. The ANOVA software was used to test whether the differences in the means between multiple data sets were statistically significant. A difference was statistically significant at $\mathrm{p}<0.05$.

\section{Results}

General data of subjects in the three groups. Age and sex were comparable in the three groups (Table I), and the differences between groups were not statistically significant $(p>0.05)$. The numbers of patients who had symptoms of fever and cough, abnormal findings in chest X-ray radiography, and positive responses in MP antibody test were comparable in the mild and severe pneumonia group, and the differences between the two groups were not statistically significant $(\mathrm{p}>0.05)$.

Levels of D-dimer and INF- $\gamma$ in healthy children and pediatric patients before treatment. As shown in Table II, the plasma D-dimer levels in the control, the mild and the severe pneumonia group were $0.08 \pm 0.19,0.30 \pm 0.21$ and $0.61 \pm 0.25 \mu \mathrm{g} / \mathrm{ml}$, respectively. Apparently, the D-dimer levels in patients with pneumonia were significantly higher than that in healthy children $(\mathrm{p}<0.001)$, and it tended to be higher in patients with severe pneumonia $(\mathrm{p}<0.001)$.

Levels of D-dimer and INF- $\gamma$ in patients with mild MPP after 24 and $120 \mathrm{~h}$ of treatment. The plasma D-dimer levels 
Table II. Levels of D-dimer and INF- $\gamma$ in healthy children and pediatric patients before treatment.

\begin{tabular}{|c|c|c|c|c|c|c|}
\hline \multirow[b]{2}{*}{ Groups } & \multicolumn{3}{|c|}{$\mathrm{D}$-dimer $(\mu \mathrm{g} / \mathrm{ml})$} & \multicolumn{3}{|c|}{$\mathrm{INF}-\gamma(\mathrm{pg} / \mathrm{ml})$} \\
\hline & Value & P-value & t-value & Value & P-value & t-value \\
\hline Control & $0.08 \pm 0.19$ & - & - & $24.01 \pm 3.17$ & - & - \\
\hline Mild pneumonia & $0.30 \pm 0.21$ & 0.0012 & 3.840 & $33.16 \pm 4.38$ & 0.003 & 3.421 \\
\hline Severe pneumonia & $0.61 \pm 0.25$ & 0.0001 & 7.235 & $46.43 \pm 4.10$ & 0.0001 & 6.595 \\
\hline
\end{tabular}

INF- $\gamma$, interferon- $\gamma$.

Table III. Levels of D-dimer and INF- $\gamma$ in patients with mild MPP over time in the treatment.

\begin{tabular}{lcc}
\hline Treatment time $(\mathrm{h})$ & D-dimer $(\mu \mathrm{g} / \mathrm{ml})$ & $\mathrm{INF}-\gamma(\mathrm{pg} / \mathrm{ml})$ \\
\hline 24 & $0.44 \pm 0.17$ & $26.15 \pm 2.09$ \\
120 & $0.19 \pm 0.09$ & $18.62 \pm 1.98$ \\
\hline
\end{tabular}

INF- $\gamma$, interferon- $\gamma$; MPP, Mycoplasma pneumoniae pneumonia.

Table IV. Levels of D-dimer and INF- $\gamma$ in patients with severe MPP over time in the treatment.

\begin{tabular}{lcc}
\hline Treatment time $(\mathrm{h})$ & D-dimer $(\mu \mathrm{g} / \mathrm{ml})$ & INF- $\gamma(\mathrm{pg} / \mathrm{ml})$ \\
\hline 24 & $0.69 \pm 0.21$ & $51.22 \pm 2.31$ \\
120 & $0.33 \pm 0.17$ & $36.71 \pm 2.08$ \\
\hline
\end{tabular}

INF- $\gamma$, interferon- $\gamma$; MPP, Mycoplasma pneumoniae pneumonia.

were $0.44 \pm 0.17$ and $0.19 \pm 0.09 \mu \mathrm{g} / \mathrm{ml}$, respectively, after 24 and $120 \mathrm{~h}$ of treatment in pediatric patients with mild MPP (Table III). Clearly, the D-dimer level decreased over time in the treatment. The decrease between the two recorded time-points was statistically significant. The INF- $\gamma$ levels were $26.15 \pm 2.09$ and $18.62 \pm 1.98 \mathrm{pg} / \mathrm{ml}$, respectively, after 24 and $120 \mathrm{~h}$ of treatment in pediatric patients with mild MPP. The decreasing trend in the INF- $\gamma$ level over time in the treatment was similar to that in the D-dimer level. The decrease between the two recorded time-points was statistically significant $(\mathrm{p}<0.05)$.

Levels of D-dimer and INF- $\gamma$ in patients with severe MPP after 24 and 120 h of treatment. The plasma D-dimer levels were $0.69 \pm 0.21$ and $0.33 \pm 0.17 \mu \mathrm{g} / \mathrm{ml}$, respectively, after 24 and $120 \mathrm{~h}$ of treatment in pediatric patients with severe MPP (Table IV). The decrease between the two time-points in the treatment was statistically significant $(\mathrm{p}<0.001)$. The INF- $\gamma$ levels were $51.22 \pm 2.31$ and $36.71 \pm 2.08 \mathrm{pg} / \mathrm{ml}$, respectively, after 24 and $120 \mathrm{~h}$ of treatment in pediatric patients with severe MPP. The decrease between the two time-points in the treatment was statistically significant $(\mathrm{p}<0.01)$. Although the decreasing trends in both the D-dimer and the INF- $\gamma$ level were expected to be the same between patients with mild and
Table V. Levels of D-dimer and INF- $\gamma$ in patients who developed extrapulmonary complications in the mild and severe pneumonia group.

\begin{tabular}{lcc}
\hline Groups & $\begin{array}{c}\text { D-dimer } \\
(\mathrm{pg} / \mathrm{ml})\end{array}$ & $\begin{array}{c}\mathrm{INF}-\gamma \\
(\mu \mathrm{g} / \mathrm{ml})\end{array}$ \\
\hline $\begin{array}{l}\text { Mild pneumonia } \\
\text { With extra pulmonary } \\
\text { complications }\end{array}$ & $0.27 \pm 0.18$ & $23.62 \pm 2.36$ \\
$\begin{array}{l}\text { Without extra pulmonary } \\
\text { complications }\end{array}$ & $0.11 \pm 0.12$ & $14.15 \pm 2.20$ \\
$\begin{array}{l}\text { Severe pneumonia } \\
\text { With extra pulmonary }\end{array}$ & $0.50 \pm 0.16$ & $41.62 \pm 2.19$ \\
$\begin{array}{l}\text { complications } \\
\text { Without extra pulmonary }\end{array}$ & $0.18 \pm 0.10$ & $33.15 \pm 2.31$ \\
complications & & \\
\hline
\end{tabular}

INF- $\gamma$, interferon- $\gamma$.

severe pneumonia, the final levels of both indicators after $120 \mathrm{~h}$ of treatment were higher in the severe pneumonia group than those in the mild pneumonia group.

Levels of D-dimer and INF- $\gamma$ in patients who developed extra-pulmonary complications in the mild and severe pneumonia group. Patients in the mild and severe pneumonia group were further divided into four subgroups: The mild pneumonia with extra-pulmonary complications subgroup, the mild pneumonia without extra-pulmonary complications subgroup, the severe pneumonia with extra-pulmonary complications subgroup, and the severe pneumonia without extra-pulmonary complications subgroup. The levels of D-dimer and INF- $\gamma$ in these four subgroups were compared after $120 \mathrm{~h}$ of treatment. Among patients in the mild pneumonia group, the D-dimer levels in patients who developed extra-pulmonary complications and those who did not were $0.27 \pm 0.18$ and $0.11 \pm 0.12 \mu \mathrm{g} / \mathrm{ml}$, respectively. The former was more than double the latter, and the difference was statistically significant $(\mathrm{p}<0.001)$. The INF- $\gamma$ levels in patients who developed extra-pulmonary complications and those who did not were $23.62 \pm 2.36$ and $14.15 \pm 2.20 \mathrm{pg} / \mathrm{ml}$, respectively. The former was much more than the latter, and the difference was statistically significant $(\mathrm{p}<0.001)$ (Table V). 


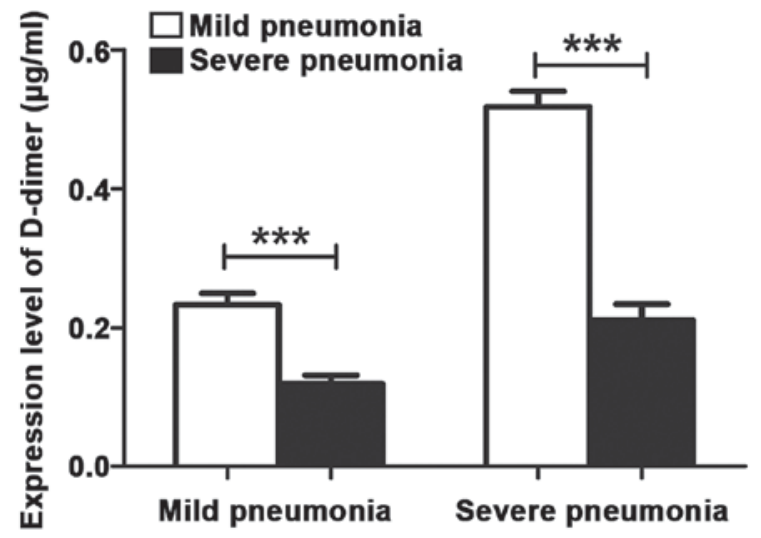

Figure 1. D-dimer levels in patients who developed extra-pulmonary complications. When ${ }^{* * *} \mathrm{P}<0.001$, the difference was significant.

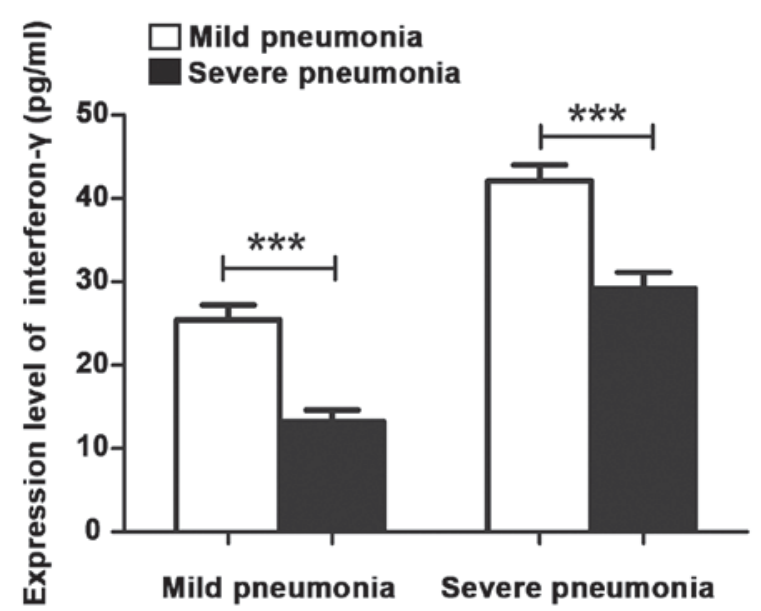

Figure 2. INF- $\gamma$ levels in patients who developed extra-pulmonary complications. INF- $\gamma$, interferon $-\gamma$. When ${ }^{* * * *} \mathrm{P}<0.001$, the difference was significant.

Among patients in the severe pneumonia group, the D-dimer levels in patients who developed extra-pulmonary complications and those who did not were $0.50 \pm 0.16$ and $0.18 \pm 0.10 \mu \mathrm{g} / \mathrm{ml}$, respectively. The former was more than double the latter, and the difference was statistically significant $(\mathrm{p}<0.001)$. The INF- $\gamma$ levels in patients who developed extra-pulmonary complications and those who did not were $41.62 \pm 2.19$ and $33.15 \pm 2.31 \mathrm{pg} / \mathrm{ml}$, respectively. The former was higher than the latter, and the difference was statistically significant $(\mathrm{p}<0.001)$ (Table V; Figs. 1 and 2).

\section{Discussion}

Pneumonia is an inflammation of the alveoli, the airways, and interstitium of the lungs caused by infection of a variety of pathogenic microorganisms or by inhaling toxic substances (9). The symptoms often include fever, cough and expectoration. For severe pneumonia, dyspnea and chest pain can also occur (10). Childhood pneumonia is a common pediatric disease with a high incidence. In most cases, the pathogens are bacteria, viruses, and MP, which inflict substantial harm to pediatric patients' lungs and systemic health. In recent years, childhood pneumonia associated with MP infection has drawn much attention from medical practitioners due to a significantly rising incidence being observed in children (11). In the clinic, it is still difficult to accurately identify childhood pneumonia associated with MP infection as the clinical symptoms are not consistent. Delayed diagnosis not only increases the risk of irreversible damage to patients' respiratory system, but also potentially leads to harm in multiple organs and systems (12). Therefore, accurate, rapid and timely identification of MPP in children is critical and should be the focus of clinical work.

It was reported that immune response to MP infection played an important role in the onset and progression of MPP. Thus, immunoassays relevant to hematology following infection with MP have become a hot topic in recent years (13). In this study, the levels of D-dimer and INF- $\gamma$ were measured in blood samples collected from 185 pediatric patients with MPP and 92 healthy children. To the best of our knowledge, there is no report in literature on assessment of these two indicators at the same time in the diagnosis and identification of MPP in children (5,14-16).

D-dimer is a specific fibrin degradation product after activation by cross-linking of the fibrin monomer with activating factors. It is also a specific marker of the fibrinolytic system. D-dimer can be used as one of the indicators for monitoring inflammation and severe infection (17). Patients with MPP appear hypoxic. Hypoxia and endotoxin stimulates inflammatory cells to release a variety of inflammatory mediators, resulting in injuries of vascular endothelial cells leading to a significant increase in plasma D-dimer levels (18).

INF- $\gamma$ is a small polypeptide that regulates cell function. It plays an important role in cell-mediated immune regulation (19). On the one hand, INF- $\gamma$ stimulates the production of macrophages. On the other hand, INF- $\gamma$ promotes the differentiation of $\mathrm{T}$ cells into cytotoxic $\mathrm{CD} 4^{+} \mathrm{TH} 1$ and $\mathrm{CD} 8^{+} \mathrm{Tc} 1 \mathrm{~T}-$-cell subsets. In addition, INF- $\gamma$ activates neutrophils and vascular endothelial cells, thereby promoting endothelial cellular secretion and adhesion (20).

In this study, 92 healthy children were recruited as a control, 90 pediatric patients with mild pneumonia and 95 pediatric patients with severe pneumonia were recruited as study subjects. The general data such as age and sex of subjects in the three groups were comparable, and the differences were not statistically significant. Blood samples were collected from all pediatric patients before and after treatment, and from healthy subjects in the control group as well. The levels of D-dimer and INF- $\gamma$ were determined for all subjects in the three groups. The results showed that the levels of D-dimer and INF- $\gamma$ in the mild and severe pneumonia group were all significantly higher than those in the control group, and the levels of both indicators increased progressively with the disease severity. Elevation of the D-dimer level implied that the pediatric patient was at the stage of MP infection, and elevation of the INF- $\gamma$ level indicated that the patient was infected and was seriously ill. After a period of treatment, the levels of D-dimer and INF- $\gamma$ were measured again in all subjects. It was found that the levels of these two indicators decreased significantly after treatment, and the final levels of both indicators after $120 \mathrm{~h}$ of treatment were higher in the severe pneumonia group than those in the mild pneumonia group. The immunoassay showed that the levels of D-dimer and INF- $\gamma$ increased significantly in pediatric patients with 
MPP, and the increase was much more evident in patients with severe pneumonia. The levels of D-dimer and INF- $\gamma$ were found to be positively correlated with extra-pulmonary complications in pediatric patients with pneumonia. The sensitivity, specificity and negative predictive value were 90.18 , 91.75 and $76.2 \%$, respectively. The sensitivity and specificity were all in the good range. In the case of severe MPP, excessive and persistent stimulation of inflammatory cytokines inflicted damage on the vascular endothelial cells, disturbing the balance between coagulation, anticoagulation and fibrinolysis. Elevation of the D-dimer level in pediatric patients with MPP indicated the presence of a hypercoagulable state and vascular endothelial dysfunction in patients, resulted from damage of vascular endothelial cells inflicted by inflammatory cytokines. When an infection occurs, early induced responses play an important role. The body secretes IFN- $\gamma$ to inhibit the growth of pathogens directly and participate in the immune response in vivo. Results of this study suggested that the serum IFN- $\gamma$ level in pediatric patients with pneumonia may be positively correlated with disease severity. The severity of the patient's condition can be evaluated by detecting the serum IFN- $\gamma$ level in pediatric patients with pneumonia. As a benefit, the patients get timely and appropriate treatment. This study demonstrated that the inflammatory response of diseases can be evaluated by assessing the levels of D-dimer and INF- $\gamma$ in plasma, which was helpful in early diagnosis of severe diseases and prediction of patient prognosis.

In summary, the levels of D-dimer and INF- $\gamma$ in pediatric patients with MPP were measured, and the results provided evidence that the assessment of both indicators at the same time may prove useful in the diagnosis of MPP in children, the evaluation of the disease severity and the prediction of prognosis. The findings in this study can serve as a reference for rapid diagnosis of MPP, evaluation of treatment efficacy and prediction of prognosis.

\section{Acknowledgements}

Not applicable.

\section{Funding}

No funding was received.

\section{Availability of data and materials}

The datasets used and/or analyzed during the present study are available from the corresponding author on reasonable request.

\section{Authors' contributions}

XJ drafted the manuscript. XJ, YZhu and YZha were mainly devoted to the sample collection and plasma D-dimer detection. JC, LR and XZ were responsible for the serum INF- $\gamma$ detection. All authors read and approved the final manuscript.

\section{Ethics approval and consent to participate}

The study was approved by the Ethics Committee of the Second Affiliated Hospital of Qiqihar Medical University
(Qiqihar, China). Guardians of all the children participating in this study signed informed consent and children voluntarily participated in this study.

\section{Patient consent for publication}

Not applicable.

\section{Competing interests}

The authors declare that they have no competing interests.

\section{References}

1. Leong CL, Norazah A, Azureen A and Lingam R: Community-acquired necrotising pneumonia caused by Panton-Valentine leucocidin-producing methicillin-resistant Staphylococcus aureus. Med J Malaysia 72: 378-379, 2017.

2. Tashiro M, Fushimi K, Kawano K, Takazono T, Saijo T, Yamamoto K, Kurihara S, Imamura Y, Miyazaki $\mathrm{T}$, Yanagihara K, et al: Adjunctive corticosteroid therapy for inpatients with Mycoplasma pneumoniae pneumonia. BMC Pulm Med 17: 219, 2017.

3. Qiu L, Wang L, Tan L, Li M, Wu C, Li L, Zhang Z, Jiang H, Sun $\mathrm{Q}$ and Zhang T: Molecular characterization of genomic DNA in Mycoplasma pneumoniae strains isolated from serious mycoplasma pneumonia cases in 2016, Yunnan, China. Infect Genet Evol 58: 125-134, 2018.

4. Millot G, Voisin B, Loiez C, Wallet F and Nseir S: The next generation of rapid point-of-care testing identification tools for ventilator-associated pneumonia. Ann Transl Med 5: 451, 2017.

5. Li YX, Zheng ZG, Liu N, Wang XN, Wu LL and Chen RC: Risk factors for pulmonary embolism in acute exacerbation of chronic obstructive pulmonary disease. Zhonghua Jie $\mathrm{He} \mathrm{He} \mathrm{Hu}$ Xi Za Zhi 39: 298-303, 2016 (In Chinese).

6. Imdad A, Mayo-Wilson E, Herzer K and Bhutta ZA: Vitamin A supplementation for preventing morbidity and mortality in children from six months to five years of age. Cochrane Database Syst Rev 3: CD008524, 2017.

7. Zhang Y, Zhou X, Zhang H, Huan C and Ye Z: Establishment of Acinetobacter baumannii-induced pneumonia model in mice. Xi Bao Yu Fen Zi Mian Yi Xue Za Zhi 33: 1392-1397, 2017 (In Chinese).

8. Miyashita N, Obase Y, Ouchi K, Kawasaki K, Kawai Y, Kobashi Y and Oka M: Clinical features of severe Mycoplasma pneumoniae pneumonia in adults admitted to an intensive care unit. J Med Microbiol 56: 1625-1629, 2007.

9. Li MS, Hung GC, Yang SY, Pan CH, Liao YT, Tsai SY, Chen CC and Kuo CJ: Excess incidence and risk factors for recurrent pneumonia in bipolar disorder. Psychiatry Clin Neurosci 72: 337-348, 2018.

10. Li W, Ding C and Yin S: Aging increases the expression of lung CINCs and MCP-1 in senile patients with pneumonia. Oncotarget 8: 108604-108609, 2017.

11. Minz A, Agarwal M, Singh JV and Singh VK: Care seeking for childhood pneumonia by rural and poor urban communities in Lucknow: A community-based cross-sectional study. J Family Med Prim Care 6: 211-217, 2017.

12. Yin YD, Wang R, Zhuo C, Wang H, Wang MG, Xie CM, She DY, Yuan X, Wang RT, Cao B, et al: Macrolide-resistant Mycoplasma pneumoniae prevalence and clinical aspects in adult patients with community-acquired pneumonia in China: A prospective multicenter surveillance study. J Thorac Dis 9: 3774-3781, 2017.

13. Joob B and Wiwanitkit V: Leukoagglutination, Mycoplasma pneumoniae pneumonia and EDTA acid blood. Turk J Haematol 35: 75-76, 2018

14. Parois SP, Faoüen A, Le Floc'h N and Prunier A: Influence of the inflammatory status of entire male pigs on their pubertal development and fat androstenone. Animal 11: 1071-1077, 2017.

15. Ye Q, Xu XJ, Shao WX, Pan YX and Chen XJ: Mycoplasma pneumoniae infection in children is a risk factor for developing allergic diseases. ScientificWorldJournal 2014: 986527, 2014.

16. Jiang YL and Peng DH: Serum level of vitamin A in children with pneumonia aged less than 3 years. Zhongguo Dang Dai Er Ke Za Zhi 18: 980-983, 2016 (In Chinese). 
17. Wu HB, Ma WG, Zhao HL, Zheng J, Li JR, Liu O and Sun LZ: Risk factors for continuous renal replacement therapy after surgical repair of type A aortic dissection. J Thorac Dis 9: 1126-1132, 2017

18. Yuan SJ, Xie HT and Li ZL: Clinical significance of hypersensitive C-reactive protein, fribrinogen and D-dimmer in connective tissue disease-related interstitial lung disease. Nan Fang Yi Ke Da Xue Xue Bao 37: 415-419, 2017 (In Chinese).

19. Duarte T, Barbisan F, do Prado-Lima PAS, Azzolin VF, da Cruz Jung IE, Duarte MMMF, Teixeira CF, Mastella MH, da Cruz IBM: Ziprasidone, a second-generation antipsychotic drug, triggers a macrophage inflammatory response in vitro. Cytokine 106: 101-107, 2018.
20. Xu J, Gu Y, Sun J, Zhu H, Lewis DF and Wang Y: Reduced CD200 expression is associated with altered Th1/Th2 cytokine production in placental trophoblasts from preeclampsia. Am J Reprod Immunol 79: e12763, 2018.

(i) (9) This work is licensed under a Creative Commons Attribution-NonCommercial-NoDerivatives 4.0 International (CC BY-NC-ND 4.0) License. 